\title{
Bifurcation Analysis of a Simplified Model of a Pressure Relief Valve Attached to a Pipe*
}

\author{
Csaba Bazsó ${ }^{\dagger}$, Alan R. Champneys ${ }^{\ddagger}$, and Csaba J. Hős ${ }^{\dagger}$
}

\begin{abstract}
A two-parameter bifurcation analysis is performed on a mathematical model that represents the motion of a pressure relief valve connected to a reservoir via a pipe. The system comprises a dimensionless system of five differential equations representing the valve position and velocity, the reservoir pressure, and the velocity and pressure components of a quarter-wave within the pipe. Two key dimensionless parameters represent the mass flow rate into the reservoir and the length of the pipe. It is found that there are two independent forms of Hopf bifurcation: a so-called valve-only instability, which involves the valve alone and occurs when the flow rate is too low, and a coupled acoustic resonance, which involves quarter-waves within the pipe, that occurs when the pipe is too long. Secondary bifurcations are also traced including curves of grazing bifurcations where the valve body first impacts with its seat. Using a mixture of simulation and numerical continuation, it is found that these instabilities interact in a complex bifurcation diagram that involves HopfHopf interaction, bistability through catastrophic grazing bifurcations, and, for long enough pipes, subcritical torus bifurcations. A particularly important discovery is a range of parameters for which the valve-only instability is subcritical, so that large amplitude impacting chaotic motion coexists with the stable equilibrium operation.
\end{abstract}

Key words. valve chatter, pressure relief valve, Neimark-Sacker (torus) bifurcation, grazing bifurcation, impacting oscillation

AMS subject classifications. 37G15, 34A12, 35B60

DOI. $10.1137 / 130922598$

1. Introduction. In recent work the last two authors [10] studied a simplified model of a pressure relief valve attached to a reservoir, finding complex nonsmooth bifurcations such as grazing and chattering for mass flow rates below that for which a Hopf bifurcation occurs. We shall refer to this form of Hopf bifurcation as a valve-only instability, as it does not involve pipe wave dynamics in its inception (although large amplitude motion arising from this instability will couple with pipe modes). In subsequent work, the authors [1] included the additional destabilizing element of a pipe that connects the valve to the reservoir, finding another form of Hopf bifurcation where the valve dynamics resonates with a quarter-wave within the pipe. For

\footnotetext{
* Received by the editors May 28, 2013; accepted for publication (in revised form) by J. Sieber December 9, 2013; published electronically DATE. This work was supported by the UK EPSRC and by the BME, Hungary. The project was also supported in the framework of the project "Talent care and cultivation in the scientific workshops of BME" by the grant TÁMOP-4.2.2.B-10/1-2010-0009 and by the UK Engineering and Physical Sciences Programme grant on "Engineering Nonlinearity."

http://www.siam.org/journals/siads/x-x/92259.html

${ }^{\dagger}$ Department of Hydrodynamic Systems, Budapest University of Technology and Economics, P.O. Box 91, 1521 Budapest, Hungary (csaba.bazso@hds.bme.hu, csaba.hos@hds.bme.hu). The third author's work was partially supported by the János Bolyai research grant of the Hungarian Academy of Sciences.

${ }^{\ddagger}$ Department of Engineering Mathematics, University of Bristol, Bristol BS8 1TR, UK (a.r.champneys@bristol. ac.uk).
} 


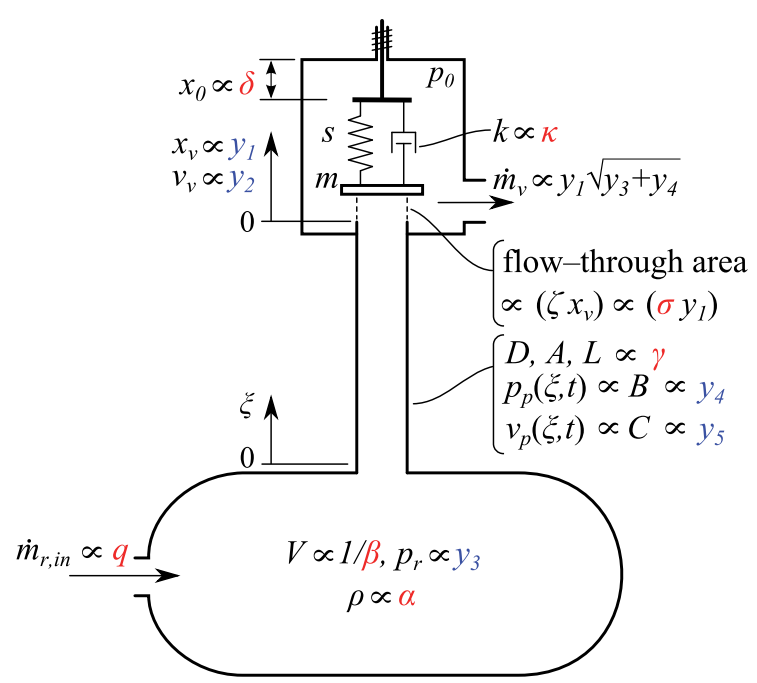

Figure 1. Schematic sketch of the mechanical model.

a given mass flow rate, this instability occurs for pipes beyond a critical length. In particular, we derived a reduced-order model that was shown to capture the nonlinear dynamics, including grazing bifurcations, sufficiently close to the initial quarter-wave instability. The purpose of the present of the paper is to study this reduced-order model in much more detail, undertaking a careful dynamical systems analysis of the combined effects of both forms of instability.

The specific configuration that we study is depicted schematically in Figure 1 and is motivated by the experimental findings announced in [13] of a dynamic instability in a conical poppet valve used for pressure relief in a hydraulic system.

The description and analysis of oscillatory instabilities in such systems goes back at least to the work of Kasai in the 1960s [11]. He produced equations of motion for a simple poppet valve and performed a linear stability analysis to find the conditions under which an oscillatory instability can occur. A similar analysis, also including the effect of a compressive reservoir tank, was carried out by MacLeod [15]. Furthermore, Kasai was able to show at least a qualitative match between the predicted instability and pressure waves observed in an experiment. Further developments of this work were carried out by a number of authors in the $80 \mathrm{~s}$ and $90 \mathrm{~s}$, culminating in the review by Hayashi [8], who also shows the propensity for impacting behavior and chaotic dynamics in a relief valve connected by a pipe to an upstream chamber (first studied by Thomann [18]). This work is further elaborated in [9], where the pipe is modeled using a single-degree-of-freedom unsteady Bernoulli equation, to arrive at a four-dimensional set of ordinary differential equations (ODEs) for the motion of the valve body. This approach was extended in a recent paper by the present authors [1], in which we solved the one-dimensional gas dynamics in the pipe using the method of characteristics. It was then demonstrated that the dominant form of instability is that due to the quarterwave frequency. It was also shown that, sufficiently close to the instability, the subsequent oscillatory motion could be captured by a single mode expansion, including only the pressure and velocity amplitude of the quarter-wave. Using a nonlinear pseudospectral (collocation) 
method, this results in two ODEs for the fluid motion in the pipe, leading to a five-dimensional system in total. It is a dimensionless version of that system which we will study in this paper.

Similar forms of oscillatory instability have been observed and analyzed in other valve systems for example, in simple plug valves [4], compressor valves [7], ball valves subject to harmonic excitation [17], pilot-operated two-stage valves [2, 19], and fluid dampers with embedded blow-off valves [6].

More recently Moussou et al. [16] conducted experiments on instabilities in a pressure relief valve attached to a water pipe for pressure that slightly exceeds the set pressure. By measuring the pressure both upstream and downstream of the valve, they were able to shed light on conditions that cause valve instability. In particular, in addition to a dynamic instability including chatter, they found evidence of a static instability that is caused by a nontrivial relationship between the valve lift and the pressure drop across the valve. They produced some preliminary linear instability analysis suggesting that the dynamic instability is due to an effective negative damping at the valve, which they postulate may couple with acoustic resonance in the pipe. A static instability was also predicted in the work of Maccari in [14], who included a continuum description of the valve and spring, allowing the effective spring constant to vary smoothly, resulting in a static fold bifurcation. Such static bifurcations are explicitly precluded in the work presented here, as we assume a simple linear spring and a trivial relationship between the valve lift and the pressure drop.

The rest of the paper is outlined as follows. In section 2, we reproduce the reducedorder model derived in [1] and introduce a suitable nondimensionalization, which leads to the identification of seven dimensionless parameters that completely specify the problem (along with additional coefficient-of-restitution and smoothing parameters). These represent the fluid compressibility, valve spring precompression, valve damping, fluid density parameter, the preset valve opening pressure, the length of the pipe, and the mass flow rate into the reservoir. Taking values for the first five of these parameters that are representative of a benchtop experimental rig using water-like fluid, the last two parameters are treated as the primary bifurcation parameters. Section 3 presents preliminary analysis of the two independent forms of Hopf bifurcation and some initial one-parameter bifurcation diagrams which reveal the nature of the dynamics beyond both instabilities. Section 4 contains the main results of the paper, in which a comprehensive two-parameter bifurcation diagram is produced using a mixture of brute force and continuation methods. Several important codimension-two points are discovered, and the dynamics in their neighborhood is studied in detail. Finally, section 5 draws conclusions, comments on practical implications of the results for operation of pressure relief valves, and suggests avenues for future research.

2. The quarter-wave model. We study a reduced-order mathematical model of the mechanical setup depicted in Figure 1, first derived in [1]. The rig consists of a pressure relief valve that vents fluid to the atmosphere, connected by a pipe of length $L$ to a reservoir of volume $V$. The model takes the form of five ODEs that describe the displacement $x_{v}(t)$ of the valve body from its seat, with corresponding velocity $v_{v}(t)$. This motion is coupled to the pressure dynamics $p_{r}(t)$ in a reservoir tank and to the pressure and velocity of a resonant acoustic wave inside the pipe. This wave is assumed to comprise only the lowest-order "organ-pipe" mode, with wavelength a quarter that of the pipe. The pressure and velocity 
amplitude of this wave are denoted by $B(t)$ and $C(t)$, respectively. As depicted in the figure, the geometric properties of the equipment are specified by constants $D, A, L$, and $V$ corresponding to the diameter, area, length of the pipe, and volume of the reservoir, respectively. The pressure relief valve is modeled as a single-degree-of-freedom oscillator characterized by its spring constant $s$, viscous damping $k$, valve body mass $m$, and spring precompression $x_{0}$. We consider compressible flow of an ideal gas with capacity heat ratio of $\hat{\kappa}$, density $\rho$, and sonic velocity $a$, while the discharge through the valve to ambient pressure $p_{0}$ is assumed to be chocked. The fluid is assumed to enter the reservoir at constant mass flow rate $\dot{m}_{r, i n}$, while the outflow rate through the valve is assumed to be a function of the valve lift $x_{v}(t)$ and the pressure at the valve end of the pipe $p_{p}(\zeta=L, t)$. Although the discharge coefficient $C_{d}$ should in general be assumed to be a function of Reynolds number, we assume fully developed turbulent flow and therefore take it to be constant. (However, see the discussion surrounding (2.3) below.)

Using the assumptions and notation of [1] - to which the reader is referred for precise meaning of all the physical parameters - the equations of motion take the form

$$
\begin{aligned}
\dot{x}_{v}= & v_{v}, \\
\dot{v}_{v}= & \frac{A}{m}\left(p_{r}+B\right)-\frac{k}{m} v_{v}-\frac{s}{m}\left(x_{0}+x_{v}\right), \\
\dot{p}_{r}= & k_{1}\left(\dot{m}_{r, i n}-\zeta x_{v} \sqrt{p_{r}+B}-k_{2} C\right), \\
\dot{B}= & -\sqrt{2} k_{1}\left(\dot{m}_{r, i n}-\zeta x_{v} \sqrt{p_{r}+B}\right)+\left(\sqrt{2} k_{1} k_{2}+k_{3} k_{4}\right) C, \\
\dot{C}= & \frac{1-\sqrt{2}}{\sqrt{2}} \frac{k_{1}}{k_{2}} \zeta^{2} x_{v}^{2}-\frac{1-\sqrt{2}}{\sqrt{2}} \frac{k_{1}}{k_{2}} \zeta \dot{m}_{r, i n} \frac{x_{v}}{\sqrt{p_{r}+B}} \\
& +\left(\frac{1-\sqrt{2}}{\sqrt{2}} k_{1} \zeta-\frac{1}{\sqrt{2}} \frac{k_{3} k_{4}}{k_{2}} \zeta\right) \frac{x_{v} C}{\sqrt{p_{r}+B}}-\sqrt{2} \frac{\zeta}{k_{2}} v_{v} \sqrt{p_{r}+B}-\frac{k_{3}}{k_{4}} B,
\end{aligned}
$$

for $x_{v}>0$, with

$$
\begin{aligned}
\zeta & =D \pi C_{d} \sqrt{\rho} \sqrt{\hat{\kappa}\left(\frac{2}{\hat{\kappa}+1}\right)^{\frac{\hat{\kappa}+1}{\hat{\kappa}-1}}} \\
k_{1} & =\frac{a^{2}}{V} \\
k_{2} & =\rho A \\
k_{3} & =2 \pi \frac{a}{4 L} \\
k_{4} & =\rho a .
\end{aligned}
$$

As is a common approximation in rigid body mechanics, we assume a Newtonian restitution impact law. While this approximation may be overly simplistic, any attempt at a more realistic law is likely to be problematic, owing to the necessity of resolving the energy dissipated by the valve and its surrounding fluid during an impact event,

$$
x_{v}\left(t^{+}\right)=0, \quad v_{v}\left(t^{+}\right)=-r v_{v}\left(t^{-}\right),
$$


where $t^{-}$and $t^{+}$denote the time just before and after the collision, respectively.

In general, the pressure $p_{r}$ should be assumed to be positive in order to drive flow through the valve. However, if the oscillatory component of pressure $B$ is large enough, then reverse flow (where $p_{r}+B<0$ ) may occur, and indeed we find that it does for sufficiently violent valve oscillations. Strictly speaking, the equations (2.1) are not valid in this case as $\sqrt{p_{r}+B}$ is not defined. In fact, the origin of this term is the relationship between pressure and flow past the valve and, for reverse flow, we need to replace this term by $-\sqrt{\left|p_{r}+B\right|}$. However, the derivative of $\pm \sqrt{\left|p_{r}+B\right|}$ becomes singular as $p_{r}+B$ approaches zero, which causes problems with numerical continuation algorithms. In fact though, $p_{r}+B \approx 0$ implies slow flow, and the assumption of fully developed turbulence breaks down. Instead, the square term should be replaced law in this case. Hence, both for reasons of physical validity and numerical convenience, in the computational results that follow, we have replaced the $\sqrt{p_{r}+B}$ term with the smooth signed square root function

$$
\operatorname{sign}\left(p_{r}+B\right) \sqrt{\left|p_{r}+B\right|} \operatorname{sgnsq}_{\varepsilon}\left(p_{r}+B\right) \equiv \tanh \left(\frac{p_{r}+B}{\varepsilon}\right) \sqrt{\left|p_{r}+B\right|} \approx\left(p_{r}+B\right) \sqrt{\left|p_{r}+B\right|},
$$

where $\varepsilon$ is a small parameter.

2.1. Nondimensionalization. The equations can be made dimensionless by rescaling with respect to a reference length scale $x_{\text {ref }}$, a reference pressure scale $p_{\text {ref }}$, and a reference frequency $\omega$ :

$$
x_{v}=x_{\text {ref }} y_{1}, \quad v_{v}=x_{\text {ref }} \omega y_{2}, \quad p_{r}=p_{\text {ref }} y_{3}, \quad B=p_{\text {ref }} y_{4}, \quad C=x_{\text {ref }} \omega y_{5},
$$

and

$$
t=\frac{\tau}{\omega}, \quad \cdot \equiv \frac{d}{d t}=\frac{1}{\omega} \frac{d}{d \tau} \equiv^{\prime} .
$$

It is convenient to make the choice

$$
p_{\text {ref }}=p_{0}, \quad x_{\text {ref }}=\frac{A p_{0}}{s}, \quad \omega=\sqrt{\frac{s}{m}} .
$$

Making the substitutions (2.4)-(2.6) into (2.1) leads to the set of dimensionless ODEs

$$
\begin{aligned}
y_{1}^{\prime}= & y_{2}, \\
y_{2}^{\prime}= & \left(y_{3}+y_{4}\right)-\kappa y_{2}-\left(\delta+y_{1}\right), \\
y_{3}^{\prime}= & \beta\left(q-y_{1} \operatorname{sgnsq}_{\epsilon}\left(y_{3}+y_{4}\right)-\frac{1}{\sigma \sqrt{2}} y_{5}\right), \\
y_{4}^{\prime}= & -\sqrt{2} \beta\left(q-y_{1} \operatorname{sgnsq}_{\epsilon}\left(y_{3}+y_{4}\right)\right)+\left(\frac{\pi}{2} \frac{\alpha}{\gamma}+\frac{\beta}{\sigma}\right) y_{5}, \\
y_{5}^{\prime}= & \left(\frac{1-\sqrt{2}}{\sqrt{2}} \beta-\sigma \frac{\pi}{2} \frac{\alpha}{\gamma}\right) \frac{y_{1} y_{5}}{\operatorname{sgnsq}_{\epsilon}}+(1-\sqrt{2}) \sigma \beta y_{1}^{2}+ \\
& -(1-\sqrt{2}) \sigma q \beta \frac{y_{1}}{\operatorname{sgnsq}_{\epsilon}\left(y_{3}+y_{4}\right)}-2 \sigma y_{2} \operatorname{sgnsq}_{\epsilon}\left(y_{3}+y_{4}\right)-\frac{\pi}{2} \frac{1}{\alpha \gamma} y_{4}
\end{aligned}
$$


Table 1

Sets of values of the dimensionless parameters used in the computations.

\begin{tabular}{lll}
\hline Quantity & Symbol & Value \\
\hline \hline Mass flow-rate & $q$ & $0-10$ \\
Pipe length parameter & $\gamma$ & $0-2.6$ \\
1/compressibility & $\beta$ & 20 \\
Spring precompression & $\delta$ & 3 \\
Damping & $\kappa$ & 1.5 \\
Density parameter & $\alpha$ & 1.3232 \\
Discharge-like parameter & $\sigma$ & 6.73346 \\
Coefficient of restitution & $r$ & 0.8 \\
Smoothing parameter of the square-root term & $\epsilon$ & 0.05 \\
\hline
\end{tabular}

for $y_{2}>0$, where the dimensionless parameters are

$$
\begin{aligned}
\kappa & =\frac{k}{m} \sqrt{\frac{m}{s}} & & \text { damping parameter, } \\
\delta & =\frac{s x_{0}}{A p_{0}} & & \text { spring precompression para } \\
\alpha & =\frac{\rho A a}{m \omega} & & \text { density parameter, } \\
\gamma & =\frac{L \omega}{a} & & \text { pipe length parameter, } \\
\sigma & =D \pi C_{d} \frac{\sqrt{p_{0}}}{\omega A \sqrt{2 \rho}} \sqrt{\hat{\kappa}\left(\frac{2}{\hat{\kappa}+1}\right)^{\frac{\hat{\kappa}+1}{\hat{\kappa}-1}}}, & & \text { discharge-like parameter, } \\
\beta & =\sqrt{2} \frac{m}{\rho V} \alpha^{2} \sigma, & & 1 / \text { compressibility, } \\
q & =\frac{\dot{m}_{r, i n} a}{p_{0} A} \frac{1}{\sqrt{2} \sigma \alpha}, & & \text { mass flow-rate parameter, } \\
\epsilon & =\frac{\varepsilon}{p_{0}} & & \text { smoothing parameter, }
\end{aligned}
$$

together with the restitution law defined by $(2.2)$ with $\left(x_{v}, v_{v}\right)$ replaced by $\left(y_{1}, y_{2}\right)$.

Parameters used in this study are typical of a laboratory-scale rig with highly incompressible (water-like) fluid; see Table 1. In what follows we shall consider the dimensionless mass flow rate $q$ and the pipe length $\gamma$ to be the primary bifurcation parameters, and hold all other parameters fixed.

\section{Preliminary analysis.}

3.1. Equilibrium solution. It is straightforward to show that for an equilibrium solution to (2.7) we must have no waves in the pipe, $y_{4}=y_{5}=0$, and no motion of the valve, $y_{2}=0$. Setting the left-hand sides of (2.7) to zero, we additionally obtain

$$
y_{3}=\left(\frac{q}{y_{1}}\right)^{2}, \quad y_{1}^{3}+\delta y_{1}^{2}-q^{2}=0 .
$$



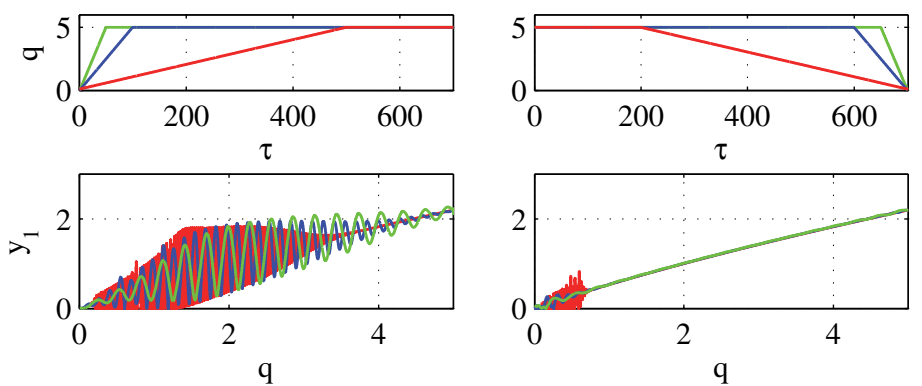

Figure 2. Simulation of a slow parameter sweep for $\gamma=0.2$. Left: Increasing the mass flow rate $q$. Right: Decreasing the mass flow rate. The rates of change in the flow rate are as follows: $d q / d t=0.01$ (red), $d q / d t=0.05$ (blue), $d q / d t=0.1$ (green). Other parameters are as in Table 1.
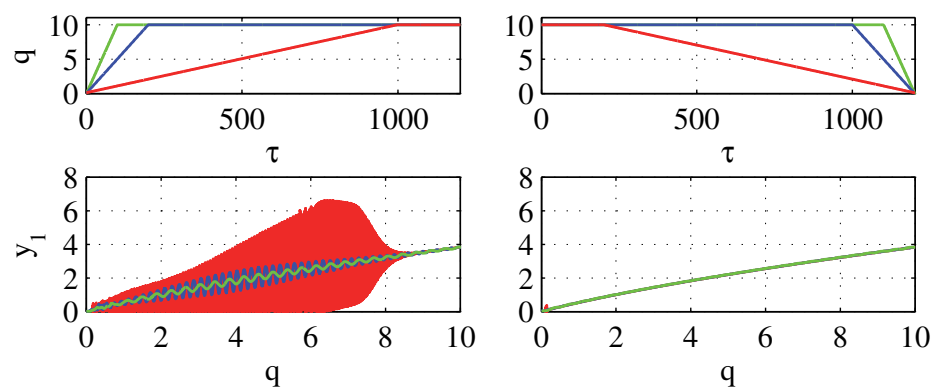

Figure 3. Similar to Figure 2 but for $\gamma=0.5$.

Note that for all $\delta \geq 0$ and $q>0$ the cubic has a unique positive solution $y_{1}^{0}$, which tends to zero as $q \rightarrow 0$. Hence there is a unique admissible equilibrium

$$
\left(y_{1}, y_{2}, y_{3}, y_{4}, y_{5}\right)=\left(y_{1}^{0}, 0, y_{3}^{0}, 0,0\right)
$$

for all parameter regions of interest, and the question remains to determine its stability.

3.2. Initial simulations. A series of transient time-domain simulations were carried out for four different pipe lengths $\gamma$, to observe what might happen in practice as the pressure relief valve either opens or closes. We found that, for each pipe length $\gamma$, there is a flow rate $q_{0}$ such that the equilibrium solution is stable for all $q>q_{0}$. We also found that if the flow rate is rapidly increased up to $q_{0}$ or reduced rapidly from $q_{0}$ to zero, then no large departure from the equilibrium is observed. However, the results in Figures 2-3 show that instabilities generally occur for low flow rates if $q$ is varied slowly.

Consider, for example, Figure 2, which is for a short pipe, $\gamma=0.2$. If the flow rate $q$ is increased sufficiently rapidly from zero (e.g., for $d q / d t=0.1$ ), although oscillations set in, they are are never of sufficient amplitude to cause the valve to impact its seat $\left(y_{1}=0\right)$. In contrast, for more gradual increase in $q$ (e.g., for $d q / d t=0.01$ ), violent impacting oscillations appear almost immediately, with growing amplitude up to $q \approx 1.4$, at which point the motion becomes impact-free. As $q$ is further increased, the amplitude decreases, until $q \approx 3.5$, where the state becomes stable. In contrast, when slowly decreasing $q$ from the stable state, instability is not lost until $q \approx 0.7$, indicating the presence of hysteresis. 
Qualitatively similar results occur for longer pipes, with in general the instability being more pronounced upon opening than on closing. Note also that the critical flow rate $q_{0}$ for stability is highly dependent on $\gamma$, with the maximum $q_{0}$ occurring for intermediate $\gamma$ values. See, for example, Figure 3 for $\gamma=0.5$, where stability is not reached upon increasing $q$ until $q \approx 8$, whereas $q_{0}$ is about 3 for $\gamma=0.2$ and about 2 for $\gamma=1.5$ (results not shown). Interestingly, even though the results are more violently unstable upon opening when $\gamma=0.5$, the pipe appears completely stable upon closing, suggesting a high degree of hysteresis in this case.

3.3. Forms of instability. It is straightforward to numerically analyze the linear stability of the equilibrium position. For each $\gamma$ we find that upon decreasing $q$, the instability occurs via a Hopf bifurcation such that the equilibrium is always unstable at sufficiently slow flow rates. There is, though, a distinction in the form of the Hopf bifurcation encountered. For low $\gamma$, the unstable eigenvector occurs primarily in the $y_{1}-y_{3}$ variables; for example, for $\gamma=0.007065, q_{\mathrm{Hopf}}=1.70078$ and the critical eigenvectors are

$$
(-0.1017 \mp 0.0652 i, 0.1766 \mp 0.2755 i, 0.9367,-0.0276 \pm 0.0119 i) .
$$

However, for higher $\gamma$, the unstable eigenvector primarily couples $\left(y_{1}, y_{2}\right)$ to $\left(y_{3}, y_{4}\right)$; for example, when $\gamma=1.5, q_{\mathrm{Hopf}}=1.30169$ and the critical eigenvectors are

$$
(0.0213 \mp 0.0386 i, 0.1179 \pm 0.0649 i, 0.0000 \mp 0.2257 i,-0.0001 \pm 0.6445 i,-0.7166) .
$$

The former kind of Hopf bifurcation can be understood as essentially a valve-only instability, as analyzed in [10] in the absence of a pipe, i.e., $\gamma=0$. The latter form of bifurcation is the quarter-wave instability that was observed in [1] and does not occur for $\gamma=0$.

To motivate why a quarter-wave instability may occur, consider the following approximate argument that is revealed by a rearrangement of the equations (2.7). The differential equations $y_{4}^{\prime}$ and $y_{5}^{\prime}$ can be rewritten in terms of reservoir pressure velocity $y_{3}^{\prime}$. After some manipulation, we obtain

$$
\begin{aligned}
& y_{1}^{\prime}=y_{2}, \\
& y_{2}^{\prime}=\left(y_{3}+y_{4}\right)-\kappa y_{2}-\left(\delta+y_{1}\right), \\
& y_{3}^{\prime}=\beta\left(q-y_{1} \sqrt{y_{3}+y_{4}}-\frac{1}{\sigma \sqrt{2}} y_{5}\right), \\
& y_{4}^{\prime}=-\sqrt{2} y_{3}^{\prime}+\frac{\pi}{2} \frac{\alpha}{\gamma} y_{5}, \\
& y_{5}^{\prime}=-2 \sigma \frac{d}{d \tau}\left(y_{1} \sqrt{y_{3}+y_{4}}\right)-\frac{\pi}{2} \frac{1}{\alpha \gamma} y_{4} .
\end{aligned}
$$

Differentiating (3.5) with respect to $\tau$ and substituting (3.4) for $y_{3}^{\prime}$ and (3.6) for $y_{5}^{\prime}$, we then obtain

$$
y_{4}^{\prime \prime}=-K_{1} \frac{d}{d \tau} Y\left(y_{1}, y_{3}, y_{4}\right)-K_{2}^{2} y_{4}, \quad \text { where } \quad Y=y_{1} \sqrt{y_{3}+y_{4}},
$$


and

$$
K_{1}=(2-\sqrt{2}) \beta+\frac{\pi \alpha \sigma}{\gamma}>0, \quad K_{2}=\sqrt{\left[\left(\frac{\pi}{2 \gamma}\right)^{2}+\frac{\beta \pi}{2 \alpha \gamma \sigma}\right]}>0 .
$$

Note that for short pipes $(\gamma \ll \pi)$ the constant $K_{2}$ can be thought of as a small adjustment to the frequency of quarter-waves in the pipe. Hence we can view (3.7) as an effective harmonic oscillator corresponding to quarter-wave oscillations in the pipe. The constant $K_{1}$ can be thought of as a coefficient of the nonlinear damping term. Hence small amplitude oscillations are damped if the nonlinear damping expression $Y^{\prime}=d Y / d \tau$ is on average proportional to $y_{4}^{\prime}$ over one cycle. Conversely, we can identify the system as being quarter-wave unstable for any parameter value for which the time average of $Y^{\prime}$ is in antiphase with $y_{4}^{\prime}$ over one cycle.

Consider $Y^{\prime}$ more carefully; assuming small changes from the equilibrium position $\left(y_{1}, y_{2}, y_{3}\right.$, $\left.y_{4}, y_{5}\right)=\left(y_{1}^{0}, 0, y_{3}^{0}, 0,0\right)$, we get

$$
\begin{aligned}
Y^{\prime} & =\frac{d}{d \tau}\left(y_{1} \sqrt{y_{3}+y_{4}}\right) \\
& =\sqrt{y_{3}+y_{4}} y_{1}^{\prime}+\frac{y_{1}}{2 \sqrt{y_{3}+y_{4}}}\left(y_{3}^{\prime}+y_{4}^{\prime}\right) \\
& \approx \sqrt{y_{3}^{0}} y_{1}^{\prime}+\frac{y_{1}^{0}}{2 \sqrt{y_{3}^{0}}}\left(y_{3}^{\prime}+y_{4}^{\prime}\right) .
\end{aligned}
$$

Now, from (3.1), for small $q$ we have

$$
y_{1}^{0} \approx \frac{q}{\sqrt{\delta}}, \quad y_{3}^{0} \approx(\delta) .
$$

Hence the first term in (3.8) dominates the second term. Intuitively then we will find instability for any coupled motion between $y_{1}$ and $y_{4}$ for which $y_{1}$ is on average in antiphase with $y_{4}$. This gives a coupled mode of instability that we should expect to dominate for small $q$.

In contrast, for large $q,(3.1)$ implies $y_{1}^{0} \approx y_{3}^{0} \approx q^{2 / 3}$. Hence the second term in (3.8) is of the same order of magnitude as the first. But this term contains an expression that is directly proportional to $y_{4}^{\prime}$, which will provide in-phase damping throughout. We might expect this term to dominate any damping that is cyclical, thus suggesting stability for higher flow rates $q$.

This approximate argument suggests that, for a finite length pipe, quarter-waves will be initially unstable for low mass flow rates $q$ but become stable for higher $q$. Hence we should expect to see a Hopf bifurcation upon reducing $q$.

4. Bifurcation analysis. We now turn to numerical bifurcation analysis to explain the above findings, delineate the parameter values at which the two forms of Hopf bifurcation occur, and try to uncover the cause of the large hysteresis we have observed in the initial simulations.

We have used AUTO [5] to trace curves of nonimpacting periodic solutions and to automatically detect and follow any fold, period-doubling, or torus bifurcations of such orbits. In addition, we have used the boundary-value solver in AUTO to automatically detect and follow grazing bifurcations of these simplest periodic orbits. We have augmented this with 


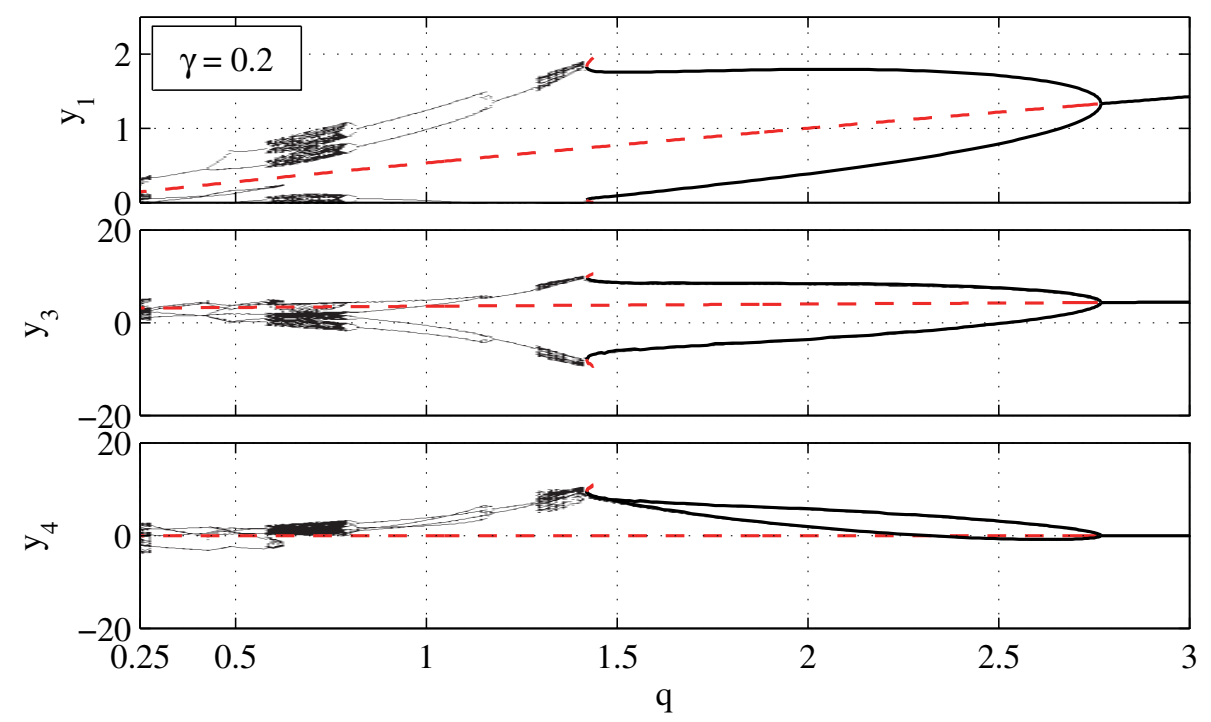

Figure 4. Bifurcation diagram for $\gamma=0.2$. In this and all subsequent plots, solid black lines show stable equilibria or simple periodic orbits computed with AUTO, with red dashed lines showing unstable orbits so computed; dots show the result of brute-force computations.

brute-force numerical bifurcation analysis using MATLAB, in which we use the dynamics at the previously computed point as the initial guess for the next parameter value and allow sufficient time for any transients to decay. Unless otherwise stated, all results are presented in the Poincaré section $y_{2}=0$.

4.1. One-parameter bifurcation diagrams. Figure 4 shows the effect of varying $q$ for $\gamma=$ 0.2. Decreasing the mass flow rate from $q=3$, the equilibrium is stable until $q=2.76845$, at which point a supercritical Hopf bifurcation occurs. Note how the initial increase in amplitude of the limit cycle is predominantly in the $y_{1}-y_{3}$ variables, showing the hallmarks of a valve-only. Nevertheless, because this is a fully coupled nonlinear system, beyond the initial instability, any finite amplitude behavior involves significant components of all phase-space variables. The limit cycle grows further in amplitude as the flow rate is further decreased until $q=1.43762$, at which point the valve first grazes with the seat. The ensuing grazing bifurcation [3], as analyzed in [13] for the case $\gamma=0$, gives rise to an impacting chaotic attractor for yet lower $q$-values. Such motion, interspersed with windows of impacting period orbits of higher period, persists all the way down to $q=0$.

Figure 5 shows the corresponding behavior for $\gamma=0.5$. The main differences here are that the initial Hopf bifurcation at $q=4.03659$ is subcritical, with the bifurcating periodic orbit restabilizing in a fold bifurcation at $q=6.92055$, before the grazing bifurcation at $q=3.6684$. There is thus a large interval of flow rates, $q \in(4.03659,6.92055)$, for which there is bistability between stable equilibrium and impacting oscillatory motion. Note that the largest portion of the impacting behavior corresponds to a period-two orbit with two impacts per period.

Figure 6 shows the corresponding bifurcation diagram for $\gamma=1.5$. Here the behavior is quite different. The first thing to note is that the initial Hopf bifurcation is once again 


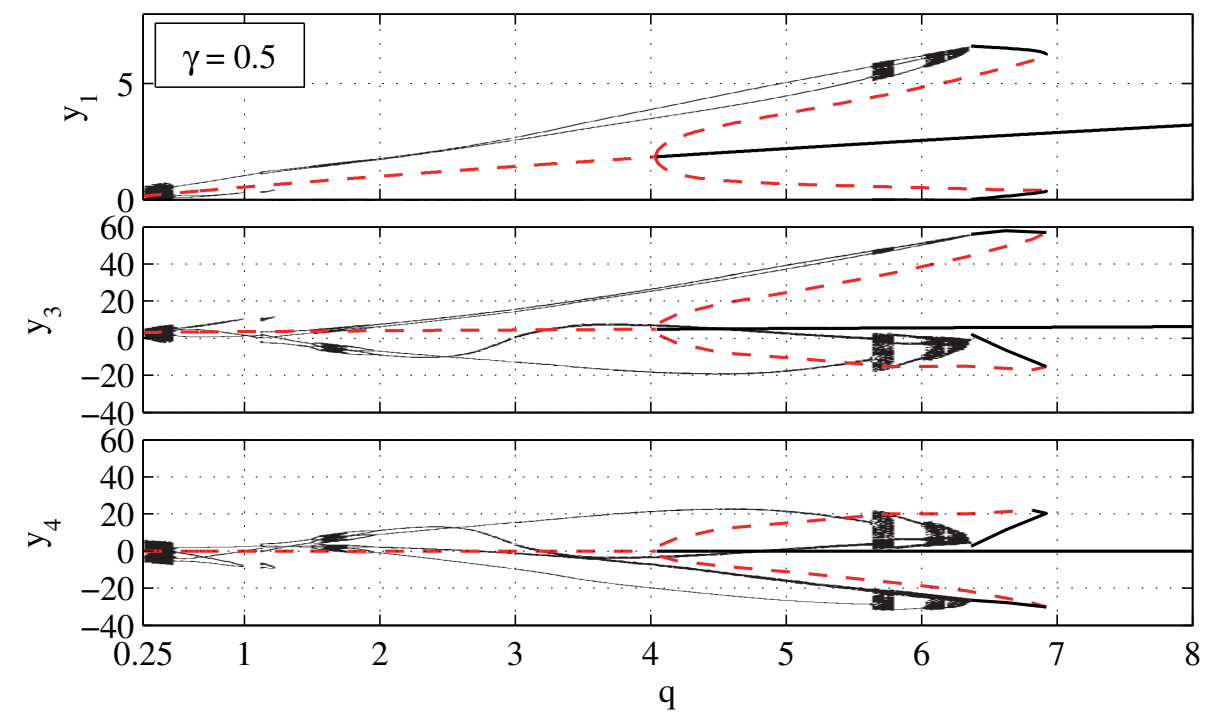

Figure 5. Similar to Figure 4 but for $\gamma=0.5$; only the $y_{1}$ component is depicted for succinctness.
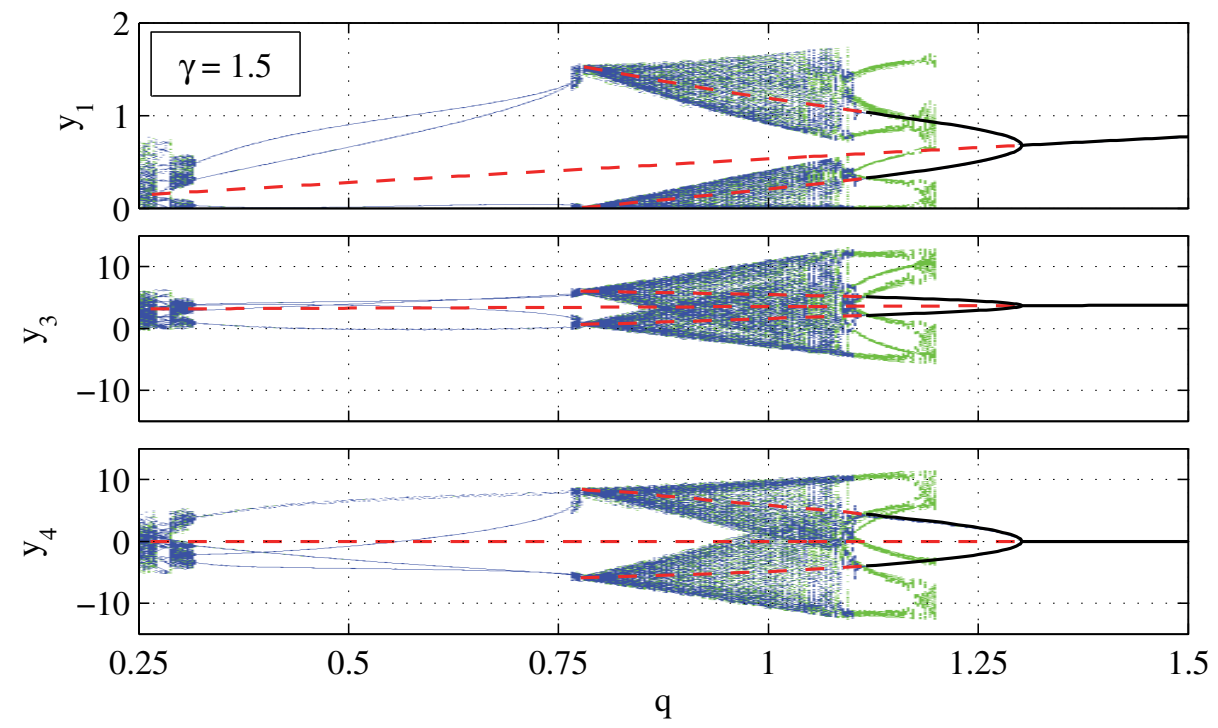

Figure 6. Similar to Figure 4 but for $\gamma=1.5$. Here blue dots represent points in the brute-force diagram obtained upon decreasing the mass flow rate $q$, while green dots represent points obtained with increasing $q$.

supercritical, and the growth in amplitude strongly couples the $\left(y_{1}, y_{2}\right)$-dynamics with that of $\left(y_{3}, y_{4}\right)$. This shows the hallmarks of a quarter-wave instability. The next thing to note is that, upon further decrease of $q$, the stable limit cycle loses stability by a new mechanism, namely a Neimark-Sacker or torus bifurcation at $q=1.11086$. The numerical bifurcation diagram strongly suggests that this torus bifurcation is actually subcritical. To see this, note that the behavior seen on decreasing $q$ is a sudden jump to what would appears to be impacting quasi-periodic behavior. (Note that the minimum $y_{1}$ value suddenly jumps to zero from about 


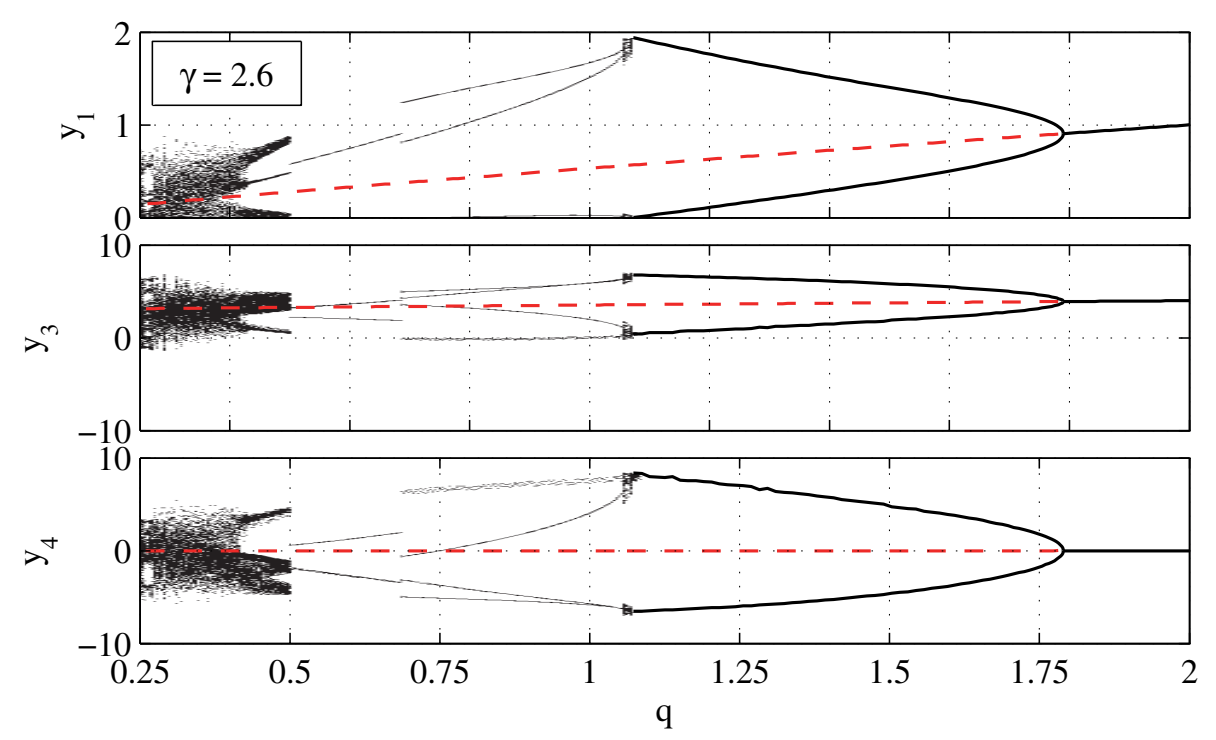

Figure 7. Similar to Figure 4, but for $\gamma=2.6$.

0.3.) Upon increasing the flow rate there is no jump near $q=1.1$, with the abrupt transition back to the stable equilibrium now occurring in what appears as a fold-like transition of an invariant torus when $q \approx 1.1985$. The motion for $q$-values just below that of the initial torus bifurcation appears to show all the hallmarks of robust chaos [3], which appears to emanate from the point at which the original, now unstable, nonimpacting period orbit undergoes a grazing bifurcation, at $q=0.76771$. The nature of the grazing bifurcation appears quite different from that happening in the previous two cases. Here the transition upon decreasing $q$ seems to be from a chaotic attractor to a period-two orbit as $q$ is decreased.

Finally, Figure 7 shows the one-parameter plot for $\gamma=2.6$. Like that for $\gamma=1.5$, the initial instability is again a supercritical quarter-wave Hopf bifurcation. But now there is no subsequent bifurcation of the stable nonimpacting periodic orbit before it loses stability through a grazing bifurcation.

4.2. Two-parameter bifurcation analysis. To explain how these bifurcations unfold in the $(q, \gamma)$ parameter plane, we have computed curves of the simplest bifurcations using AUTO. The results are presented in Figure 8 with successive zooms in Figures 9, 10, and 12.

Note the distinctive shape of the two different Hopf bifurcation curves. The quarter-wave instability arises from $q=\gamma=0$, whereas the valve-only instability occurs for finite $q$ even when $\gamma=0$. The $q$-value of the instability initially increases with $\gamma$ before disappearing altogether for $\gamma>1.365$. Also note that whereas the quarter-wave bifurcation is always supercritical, the valve instability is subcritical for an intermediate range of $\gamma$-values - between 0.3771 and 0.7691 . These two $\gamma$-values correspond to the two Bautin codimension-two points (see, e.g., [12]) $A^{*}$ and $B^{*}$, which give rise to extra curves of fold bifurcations. These folds undergo a complex sequence of other bifurcations involving codimension-two cusp points and points where a fold and a grazing occur simultaneously.

Figure 10 shows a zoom of the two-parameter plot close to the lower Bautin point $A^{*}$, and 


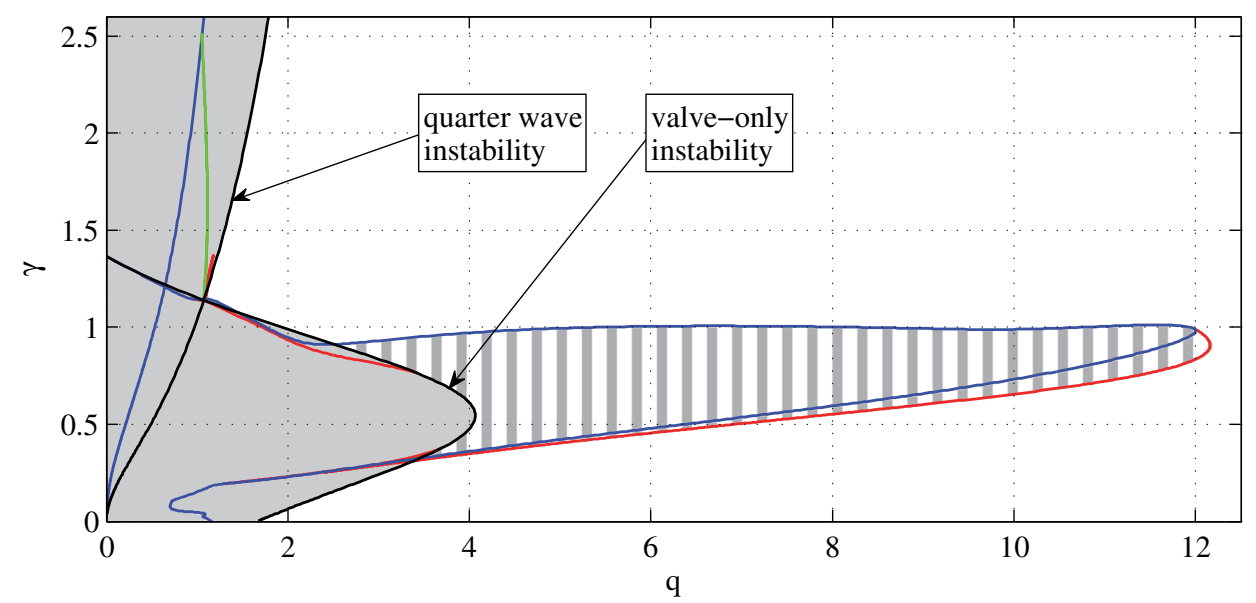

Figure 8. Two-parameter bifurcation diagram. Black curves represent location of Hopf bifurcations corresponding to quarter-wave and valve-only instabilities. Other curves represent the location of periodic orbit bifurcations; fold (red), torus bifurcation (green), and grazing bifurcation (blue). Continuous shading represents where the equilibrium is unstable, and hashed shading to where there is bistability between the equilibrium and oscillatory behavior of one kind or another.

Figure 11 illustrates the implication of the various bifurcation curves on the one-parameter bifurcation diagrams. For $\gamma=0.31$, upon decreasing the mass flow rate, stability is lost via a supercritical Hopf bifurcation at $q=3.37877$. As the stable limit cycle reaches a fold point at $q=3.16988$, the limit cycle turns back and becomes unstable until a second fold at $q=3.35226$. Here the limit cycle regains stability until it undergoes a grazing at $q=3.22192$, causing chaotic motion. For a slightly shorter pipe (not depicted) the two folds of the periodic orbit annihilate each other at the cusp point. For a slightly longer pipe, such as $\gamma=0.35$ depicted, the boundary of loss of stability and the fold points move towards higher $q$, and the unstable portion of the limit cycle branch expands. By the time $\gamma=0.3771$, the Hopf bifurcation has become subcritical.

4.3. Dynamics close to the Hopf-Hopf point. At the point $C^{*}$, where $(\gamma, q)=(1.07286$, 1.13934), the two Hopf bifurcations coincide in a codimension-two Hopf-Hopf bifurcation. The dynamics close to such codimension-two points is described in full in [12, Chap. 8] using normal for theory, including the identification of many different subcases. The left-hand plot of Figure 12 shows a zoom of the numerically computed bifurcation diagram close to $C^{*}$, in which a bifurcating curve of torus bifurcations can be clearly observed. More details in the righthand further zoom show that there are in fact two independent Niemark-Sacker bifurcations that emanate from the codimension-two point, which is one of the generic possibilities in the unfolded normal form. At first sight, from Figure 12(left), it would seem that additional fold bifurcations of periodic orbits are also involved in the codimension-two point, which would represent a degeneracy from the generic cases analysed in [12]. However, the further zoom indicate a that these bifurcations do not pass through the codimension-two point, but in fact form a separate arrangement involving two cusp points that happen to be close to the codimension-two point. To further complicate matters, the grazing bifurcation is also nearby. 


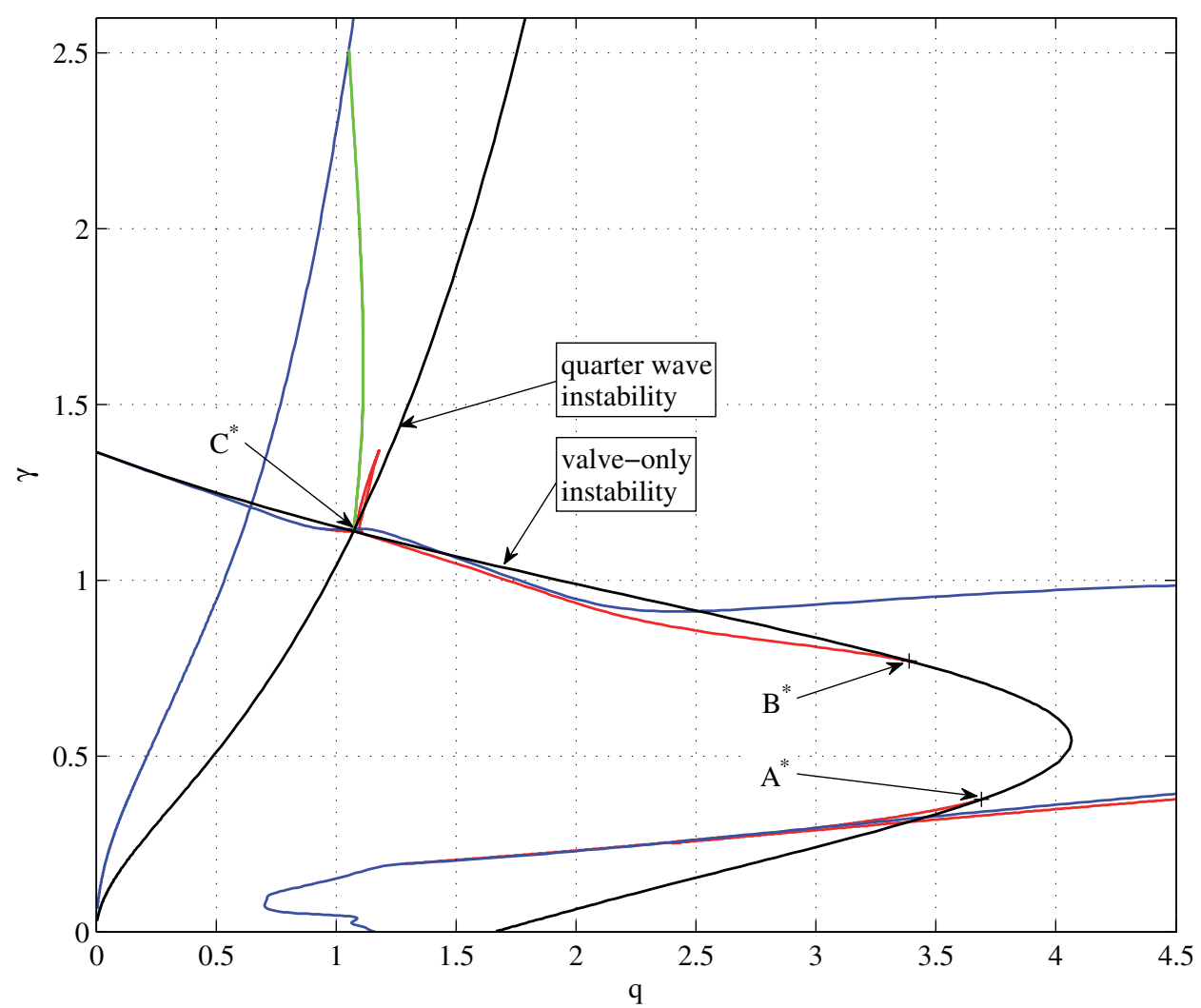

Figure 9. More detail of the bifurcation curves in Figure 8 for smaller $q$, indicating the location of three codimension-two points: a Hopf-Hopf point at $C^{*}$ and two degenerate Hopf (Bautin) bifurcations at $A^{*}$ and $B^{*}$.

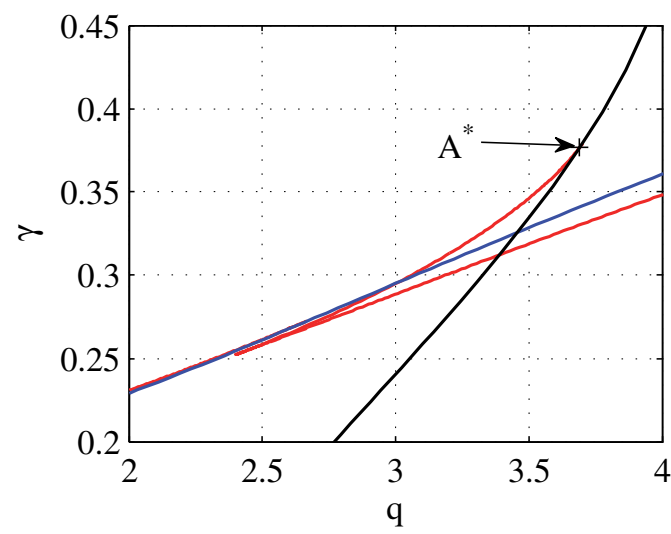

Figure 10. A zoom of Figure 8 in the region $A^{*}$.

In fact, although close in parameter space, the fold and grazing bifurcations are some distance apart in phase space.

To understand the effect on the dynamics of the complex arrangement of bifurcation curves 

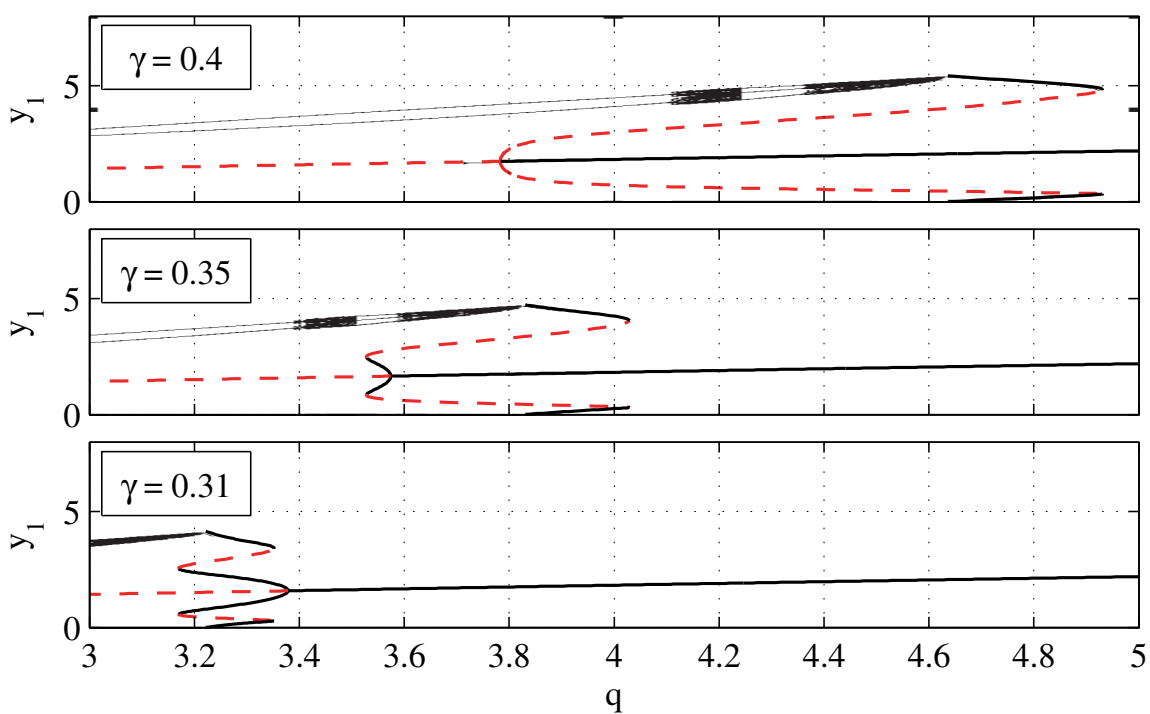

Figure 11. Evolution of one-parameter bifurcation diagrams close to the Bautin point $A^{*}$.
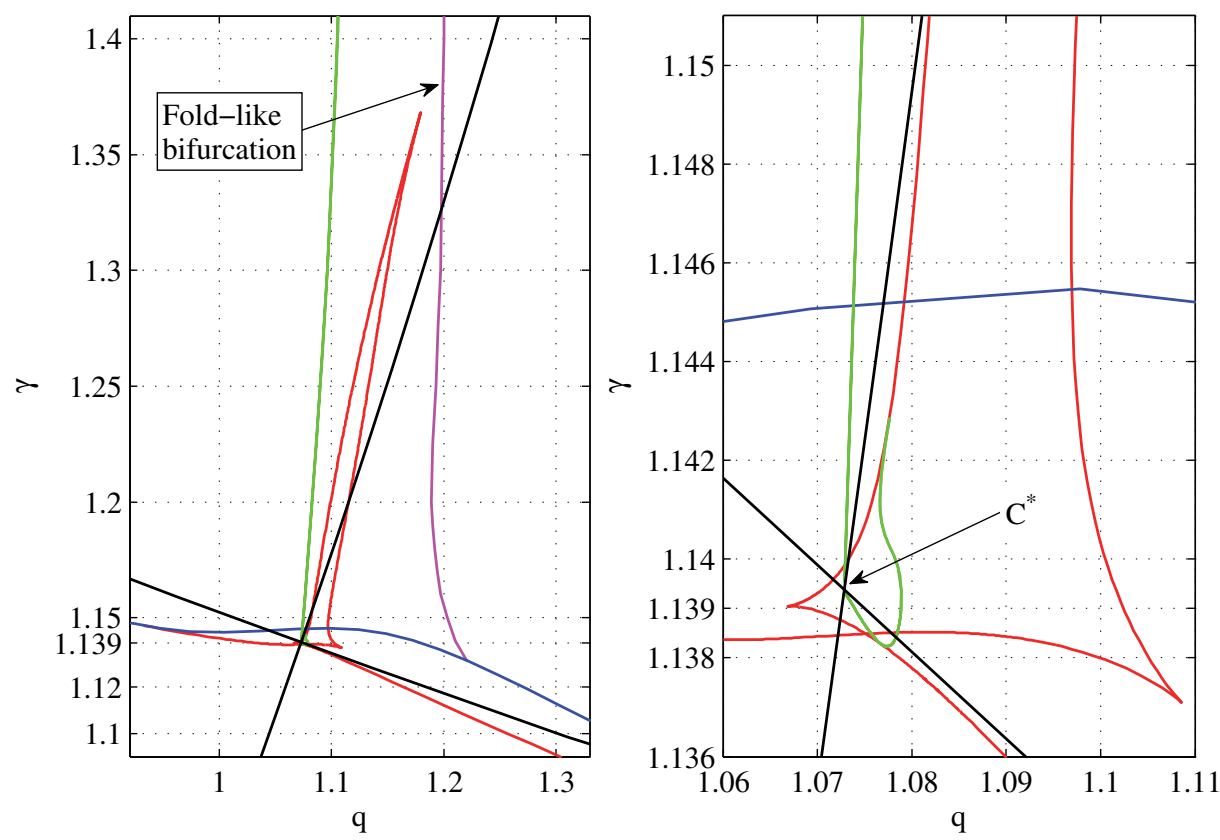

Figure 12. (Left) Zoom of Figure 8 close the Hopf-Hopf bifurcation point $C^{*}$. The color coding of curves is the same as that used there, but in addition a magenta curve indicates a fold-like bifurcation of an invariant torus. (Right) A yet further zoom, showing the complex arrangement of fold and torus bifurcation curves.

close to $C^{*}$, it is instructive to look at a sequence of one-parameter bifurcation diagrams; see Figure 13. For $\gamma=1.15$, upon decreasing the mass flow rate, the stable equilibrium loses its stability at $q=1.06913$ via a supercritical Hopf bifurcation, and the limit cycle steadily 

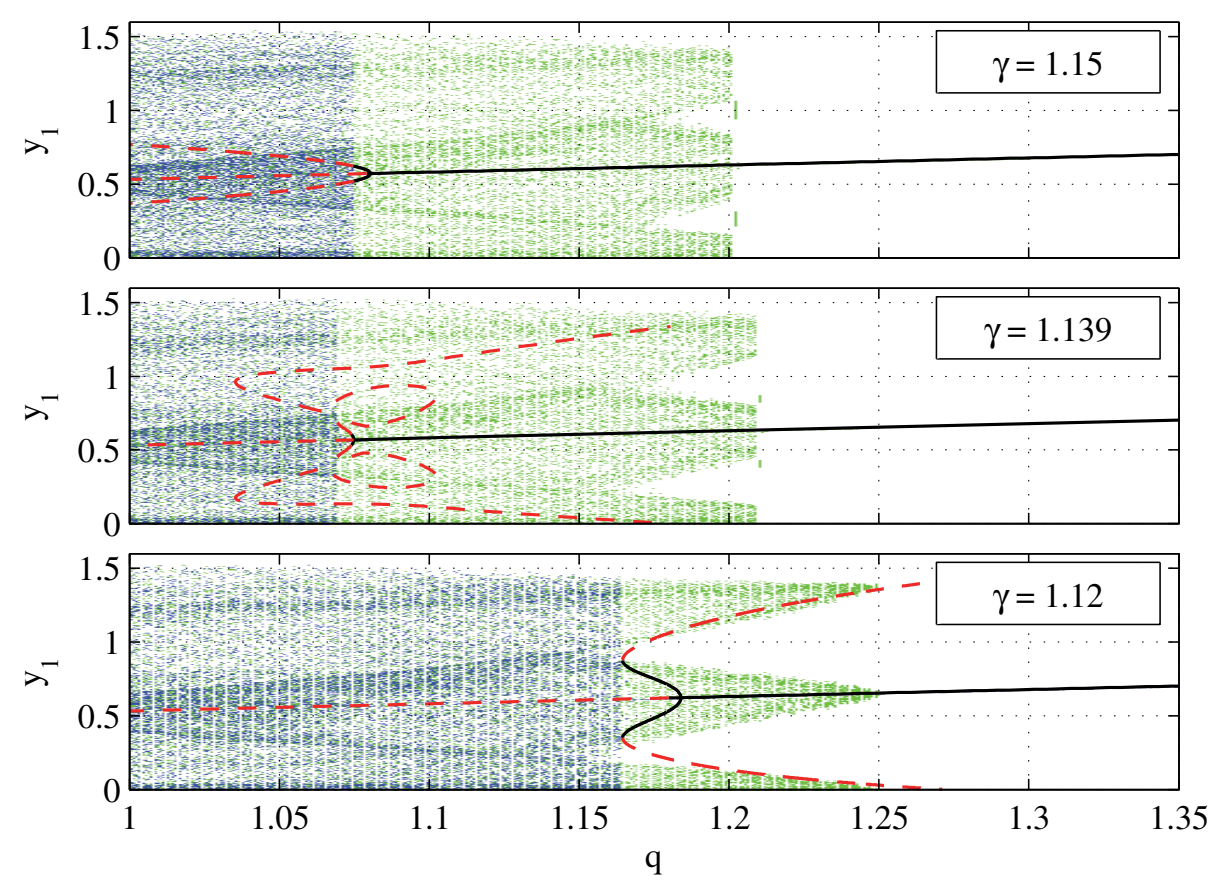

Figure 13. Evolution of one-parameter bifurcation diagrams close to the Hopf-Hopf point $C^{*}$. Here blue dots represent points in the brute force diagram obtained upon decreasing the mass flow rate, while green dots represent points obtained with increasing mass flow rate.

grows until $q=1.07458$ where the motion destabilizes in a subcritical torus bifurcation. The motion immediately jumps to large amplitude apparently quasi-periodic impacting motion. In contrast, upon increasing the mass flow rate from $q=1$, the impacting quasi-periodic motion survives until $q \approx 1.201$. At this value there seems to be a fold-like bifurcation that destroys the large amplitude behavior. We have not analyzed the precise nature of this bifurcation, but it seems likely to be the result of a grazing bifurcation of an invariant torus; see, for example, [3, Chap. 9] and references therein.

For $\gamma=1.139$ one can observe a much more complicated development of the limit cycle. The stability is lost via a supercritical Hopf bifurcation at $q=1.07481$; then the stable limit undergoes a torus bifurcation at $q=1.07403$ and turns back at a fold point $(q=1.06904)$. The limit cycle undergoes another torus bifurcation at $q=1.07887$ that is followed by two additional fold points at $q=1.10243$ and $q=1.03538$. The limit cycle grows until $q=1.18049$, where the motion is interrupted by a grazing bifurcation. The figure also indicates similar hysteresis between the stable state and the impacting quasi-periodic motion.

Finally for $\gamma=1.12$ we see yet different behavior. The Hopf bifurcation is once again supercritical. The bifurcating periodic orbit now loses stability via a fold bifurcation, and the fold-like bifurcation of the invariant torus is now replaced by a fold-like grazing bifurcation of the periodic orbit.

5. Conclusion. This paper has provided a concrete example of the interaction between a Hopf-Hopf bifurcation and grazing bifurcations in a simple, physically motivated system. From 
a dynamical systems point of view, this appears to be a rich source of interesting behavior including a complex sequence of folds, and grazing and (subcritical) torus bifurcations.

It is also interesting to speculate on potential physical applicability of these results to the operation of pressure relief valves. The upper right of the parameter plane depicted in Figure 8 (large $q$ and $\gamma$ ) represents the desired operating regime for any valve. One might imagine that the fact that instability always occurs for sufficiently small flow rate might cause problems in practice, because when a pressure-relief valve first opens, $q=0$ by definition. However, as revealed in the transient computations reported in section 3 , if the transitions through low- $q$ regimes are rapid enough, no instability is observed. In practice, pressure relief valves usually open for a higher pressure than that at which they are designed to close. This means that the initial opening needs to result in a steady mass flow rate that is large enough to avoid instability.

One of the key conclusions that arises from our results is that straightforward analysis of the linearization around the equilibrium point will be insufficient to determine the region in which stability can be guaranteed. This is because of the subcritical Hopf bifurcations that occur for intermediate pipe lengths, which give rise to large hysteresis regions (light shaded in Figure 8) in which a sudden disturbance can push an otherwise stable valve into, often violent, oscillation.

Care need also be taken to avoid pipe lengths $\gamma$ that might exacerbate instability. The problem here is there appears to be no simple rule of thumb that can be applied. If the pipe is too short $(\gamma<1.365$ for the parameter set considered here), then the system is susceptible to valve-only instabilities. Yet, the longer the pipe is, the greater is the propensity for quarterwave instabilities.

As shown in [1], the model we have used is capable of capturing the true dynamics of more complex models, but only provided that the amplitude of the quarter-wave terms is not too large. In reality, then, few practical conclusions should be drawn from the motion predicted in this simplified model beyond the first grazing instability.

It should also be pointed out that the particular results presented here are valid only for the set of parameter values chosen, although preliminary searches of other physically motivated parameter regimes suggest the results to be typical for laboratory-scale systems involving water-like fluid. Although the model is capable of capturing more highly compressive fluids, the parameter values for gas-like valves would be rather different, with $\beta$ typically being five orders of magnitude smaller. Preliminary analysis has suggested that such systems are indeed susceptible to quarter-wave instability, but not to valve-only instability, making their two-parameter bifurcation diagrams somewhat simpler.

A key question that remains to be addressed is whether the model presented here matches what is observed in practice. Preliminary experimental findings are encouraging but will be presented in detail elsewhere. Indeed, the main purpose of this study has not been to match experimental data but to reveal the dynamical complexity in this simple, yet technologically important, mechanical system.

\section{REFERENCES}

[1] C. Bazsó, A.R. Champneys, And C.J. Hős, Model Reduction of a Direct Spring-Loaded Pressure Relief 
Valve with Upstream Pipe, preprint, 2013.

[2] K.K. Botros, G.H. Dunn, And J.A. HrycyK, Riser-relief valve dynamic interactions, J. Fluids Engrg., 119 (1997), pp. 671-679.

[3] M. di Bernardo, C.J. Budd, A.R. Champneys, and P. Kowalczyk, Piecewise-Smooth Dynamical Systems: Theory and Applications, Vol. 163, Springer, New York, 2007.

[4] W. D'Netto And D.S. Weaver, Divergence and limit cycle oscillations in valves operating at small openings, J. Fluids Structures, 1 (1987), pp. 3-18.

[5] E.J. Doedel, A.R. Champneys, F. Dercole, T. Fairgrieve, Yu. Kuznetsov, B. Oldeman, R. Paffenroth, B. Sandstede, X. Wang, and C. Zhang, Auto: Software for Continuation and Bifurcation Problems in Ordinary Differential Equations, technical report, Concordia University, Montreal, QC, 2009.

[6] R.D. Eyres, P.T. Pirroinen, A.R. Champneys, and N.A.J. Lieven, Grazing bifurcations and chaos in the dynamics of a hydraulic damper with relief valves, SIAM J. Appl. Dyn. Syst., 4 (2005), pp. $1076-$ 1106.

[7] R.A. Habing AND M. Peters, An experimental method for validating compressor valve vibration theory, J. Fluids Structures, 22 (2006), pp. 683-697.

[8] S. HAYAShi, Instability of poppet valve circuit, JSME Internat. J. Ser. C, 38 (1995), pp. 357-366.

[9] S. Hayashi, T. Hayase, And T. Kurahashi, Chaos in a hydraulic control valve, J. Fluids Structures, 11 (1997), pp. 693-716.

[10] C.J. Hös And A.R. Champneys, Grazing bifurcations and chatter in a pressure relief valve model, Phys. D, 241 (2012), pp. 2068-2076.

[11] K. KASAI, On the stability of a poppet valve with an elastic support: 1st report, considering the effect of the inlet piping system, Bull. JSME, 11 (1968), pp. 1068-1083.

[12] Yu.A. Kuznetsov, Elements of Applied Bifurcation Theory, Springer-Verlag, Berlin, 2004.

[13] G. Licskó, A.R. Champneys, And C.J. Hös, Nonlinear analysis of a single stage pressure relief valve, IAENG Int. J. Appl. Math., 39 (2009), pp. 1-14.

[14] A. Maccari, Saddle-node bifurcations of cycles in a relief valve, Nonlinear Dynam., 22 (2000), pp. 225247.

[15] G. MACLEOD, Safety valve dynamic instability: An analysis of chatter, J. Pressure Vessel Tech., 107 (1985), pp. 172-177.

[16] P. Moussou, R.J. Gibert, G. Brasseur, C. Teygeman, J. Ferrari, and J.F. Rit, Instability of pressure relief valves in water pipes, J. Pressure Vessel Tech., 132 (2010).

[17] A.H. NAYFeh AND H. Bouguerra, Non-linear response of a fluid valve, Internat. J. Non-Linear Mech., 25 (1990), pp. 433-449.

[18] H. Thomann, Oscillations of a simple valve connected to a pipe, Z. Angew. Math. Phys., 27 (1976), pp. 23-40.

[19] Q. Ye And J. Chen, Dynamic analysis of a pilot-operated two-stage solenoid valve used in pneumatic system, Simul. Model. Practice and Theory, 17 (2009), pp. 794-816. 